\title{
The Effect of Urinary Trypsin Inhibitor Against Neuropathic Pain in Rat Models
}

Department of Anesthesiology and Pain Medicine, Chosun University School of Medicine, *Medical School, Chonnam National University, Gwangju, Korea

Ki Tae Jung, MD, Hyun Young Lee, MD, Myung Ha Yoon, MD*, and Kyung Joon Lim, MD

\section{Background:}

Nerve injury sometimes leads to chronic neuropathic pain associated with neuroinflammation in the nervous system. In the case of chronic neuropathic pain, the inflammatory and algesic mediators become predominant and result in pain hypersensitivity following nervous system damage. It is well known that urinary trypsin inhibitor (ulinastatin, UTI) has an anti-inflammatory activity. Recently, the neuroprotective action of UTI on the nervous system after ischemic injury has been reported. Thus, we evaluated the neuroprotective effect of ulinastatin in a rat model of neuropathic pain.

\section{Methods:}

Neuropathic pain was induced with L5 spinal nerve ligation (SNL) in male Sprague-Dawley rats weighing $100-120 \mathrm{~g}$. The rats were divided into 3 groups, with $\mathrm{n}=8$ in each group. The rats in the control group (group 1) were administered normal saline and those in group 2 were administered UTI (50,000 U/kg) intravenously through the tail vein for 3 days from the day of SNL. Rats in group 3 were administered UTI $(50,000 \mathrm{U} / \mathrm{kg})$ intravenously from the $5^{\text {th }}$ day after SNL. The paw withdrawal threshold was measured using the von Frey test for 3 days starting from the $5^{\text {th }}$ day after SNL.

\section{Results:}

The paw withdrawal thresholds were significantly increased in the rats of group 2 compared to the other groups $(P<0.05)$.

\section{Conclusions:}

Ulinastatin, which was administered for 3 days after SNL, increased the paw withdrawal threshold and it could have a neuroprotective effect in the rat model of neuropathic pain. (Korean J Pain 2013; 26: 356-360)

\section{Key Words:}

neuropathic pain, rats, urinary trypsin inhibitor.

Received September 12, 2013. Revised September 17, 2013. Accepted September 21, 2013.

Correspondence to: Kyung Joon Lim, MD

Department of Anesthesiology and Pain Medicine, Chosun University School of Medicine, 365 Pilmun-daero, Dong-gu, Gwangju 501-717, Korea

Tel: +82-62-220-3223, Fax: +82-62-223-2333, E-mail: kjlim@chosun.ac.kr

(a) This is an open-access article distributed under the terms of the Creative Commons Attribution Non-Commercial License (http:// creativecommons.org/licenses/by-nc/3.0/), which permits unrestricted non-commercial use, distribution, and reproduction in any medium, provided the original work is properly cited.

Copyright (c) The Korean Pain Society, 2013 


\section{INTRODUCTION}

Neuropathic pain syndrome (NPS) can occur due to numerous causes, such as nerve damage caused by tumors, trauma, diabetic neuropathy, herpes zoster and so on. Recent studies showed that microglial activation has an important role in the generation and progress of neuropathic pain [1]. When nerve damage occurs, an immunelike glial response occurs and enhances neuronal nociceptive transmission [1]. This enhances the production of various inflammatory factors including pro-inflammatory cytokines such as interleukin (IL)-1, IL-6, IL-10, and tumor necrosis factor (TNF) [1-3]. Such cytokines may have important roles in the inflammation of the nervous system and in the development of neuropathic pain; thus, reducing pro-inflammatory cytokines with anti-inflammatory drugs could attenuate the development of NPS and decrease pain [1-3].

Ulinastatin, a urinary trypsin inhibitor (UTI), is a protease inhibitor which is purified from human urine [4]. It has an anti-inflammatory effect through the inhibition of inflammatory factors by suppressing polymorphonuclear leukocyte elastase, TNF- $\alpha$, and IL-6 [4]. Such anti-inflammatory effect has been known to produce the protection of cells in the case of organ injury $[5,6]$. Recently, it has been reported that UTI has a neuroprotective effect against cerebral injury $[7,8]$. However, there are still no studies on the protective effect of UTI on peripheral neuropathy caused by spinal nerve injury. In this study, we observed a difference in the paw withdrawal threshold to mechanical stimuli after spinal nerve ligation (SNL) when UTI was administered before or after neuropathic pain was evoked in rats. This result led us to the hypothesis that the anti-inflammatory effect of UTI can protect against inflammation caused by spinal nerve injury.

\section{MATERIALS AND METHODS}

After receiving approval from the Institutional Animal Care Committee, Research Institute of Medical Science, Sprague-Dawley male rats, weighing 100-200 g, were used for the experiments. All the rats were raised in cages under ideal conditions, kept at room temperature between $20-23^{\circ} \mathrm{C}$ with food and water available ad libitum and on a $12 \mathrm{~h}: 12 \mathrm{~h}$ night : day cycle for adaptation to the laboratory environment for one week.

The rats were anesthetized with sevoflurane, and spinal nerve ligation (SNL) was performed to induce the neuropathic pain model in rats $[9,10]$. A skin incision was made in the midline of the L5-S2 spine in the prone position and the left paraspinal muscles were separated from the spinous process. After dissection, the transverse process of the L6 spine was removed with a small rongeur to isolate the left L5 and L6 spinal nerves. Each nerve was tightly ligated with 6-O silk suture distal to the dorsal root ganglia and the wound was closed. After the rats recovered from the anesthesia, signs of L4 spinal nerve damage in the rats such as dragging of the left hind limb were observed to exclude them from the study.

The rats were divided into three groups: the control

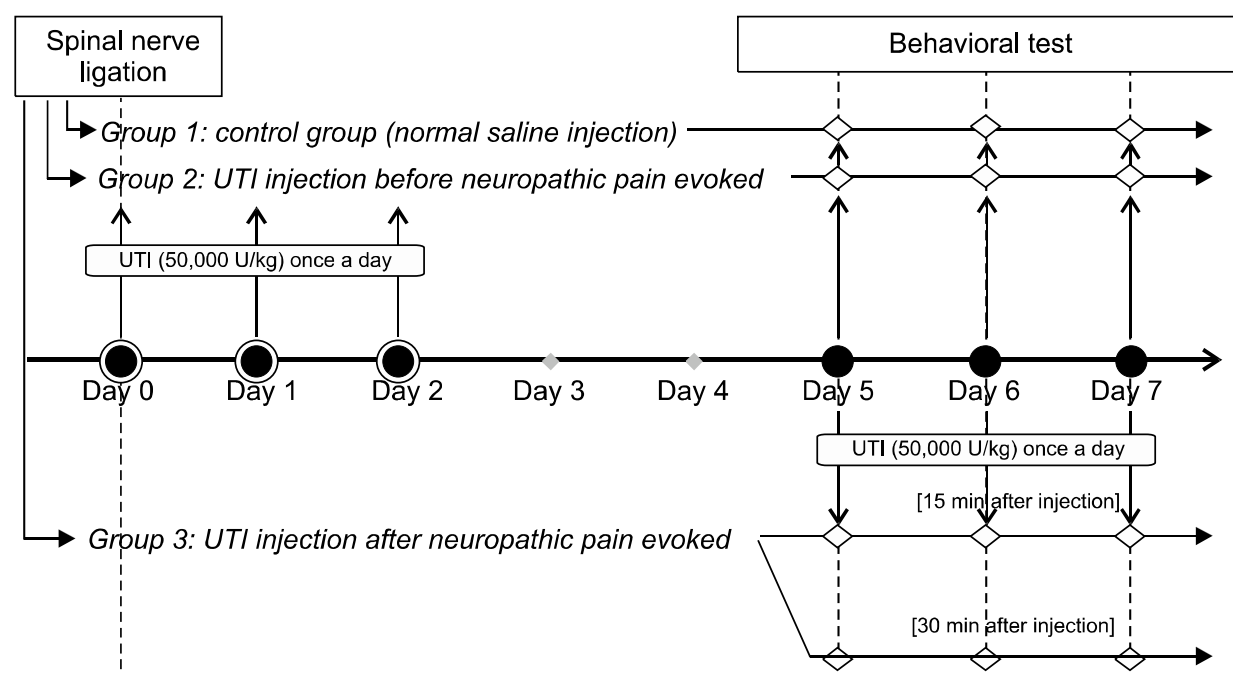

Fig. 1. Schematic diagram of study design. UTI: urinary trypsin inhibitor. 
group (group 1) who were administered normal saline for 3 days after SNL; UTI 50,000 U/kg administered before neuropathic pain was evoked (group 2); and UTI 50,000 $\mathrm{U} / \mathrm{kg}$ administered after neuropathic pain was evoked (group 3), with $\mathrm{n}=8$ for each group (Fig. 1). In group 2, $50,000 \mathrm{U} / \mathrm{kg}$ UTI (Ulistin ${ }^{\circledR}$, Hanlim pharm co., Seoul, Korea) was administered through the tail vein right after SNL for 3 days and mechanical allodynia was assessed starting from the $5^{\text {th }}$ day after SNL for 3 days. In group 3, $50,000 \mathrm{U} / \mathrm{kg}$ UTI was administered and the behavioral test was done starting from the $5^{\text {th }}$ day after SNL for 3 days. Mechanical allodynia was assessed 3 times in one day: before UTI injection, 15 minutes after UTI injection, and 30 minutes after UTI injection.

The mechanical allodynia was assessed with the paw withdrawal threshold starting from the $5^{\text {th }}$ day after spinal nerve ligation [9]. After acclimation to the test chamber for $20 \mathrm{~min}$, mechanical stimulation was applied using a

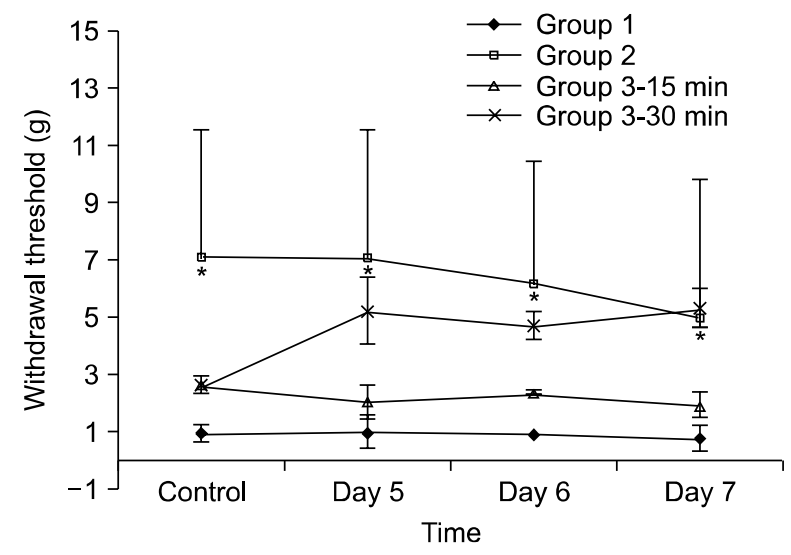

Fig. 2. Withdrawal thresholds of paw in rats were observed for 3 days from $5^{\text {th }}$ day after spinal nerve ligation. The withdrawal thresholds were significantly increased in the rats of group 2 than those of group $1(P<0.05)$. ${ }^{*} P<$ 0.05 compared with the group 1. Group 1, Sham group; Group 2, UTI 50,000 U/ kg administration from the day of SNL for 3 days before neuropathic pain has evoked; Group 3-15 min, withdrawal thresholds were assessed 15 minutes after UTI injection, UTI 50,000 U/kg administration was started from the $5^{\text {th }}$ day after SNL, which is after neuropathic pain has evoked; Group 3-30 min, withdrawal thresholds were assessed 30 minutes after UTI injection, UTI $50,000 \mathrm{U} / \mathrm{kg}$ administration was started from the $5^{\text {th }}$ day after SNL, which is after neuropathic pain has evoked; Day $5,5^{\text {th }}$ day after spinal nerve ligation; Day $6,6^{\text {th }}$ day after spinal nerve ligation; Day $7,7^{\text {th }}$ day after spinal nerve ligation. Values are mean $\pm S D$ of withdrawal threshold. series of von Frey filaments. Filaments were applied vertically to the plantar surface of the hindpaw for 5 seconds and abrupt withdrawal or flinching was considered a positive response.

All data are expressed as the mean \pm standard deviation. The percentage of maximal possible effect (\%MPE) was calculated according to the following formula [11]:

$\% M P E=\frac{\text { Postdrug threshold }- \text { baseline threshold }}{\text { Cut-off threshold(15) - baseline threshold }} \times 100$

Behavioral experiments, which were nonparametrically distributed, were analyzed by the Kruskal-Wallis test for between-group comparisons, as well as the Mann-Whitney $\mathrm{U}$ test for comparisons between pairs of groups. Values with $P<0.05$ were considered statistically significant.

\section{RESULTS}

Spinal nerve ligation produced mechanical allodynia and reduced the withdrawal threshold of the left hind paw

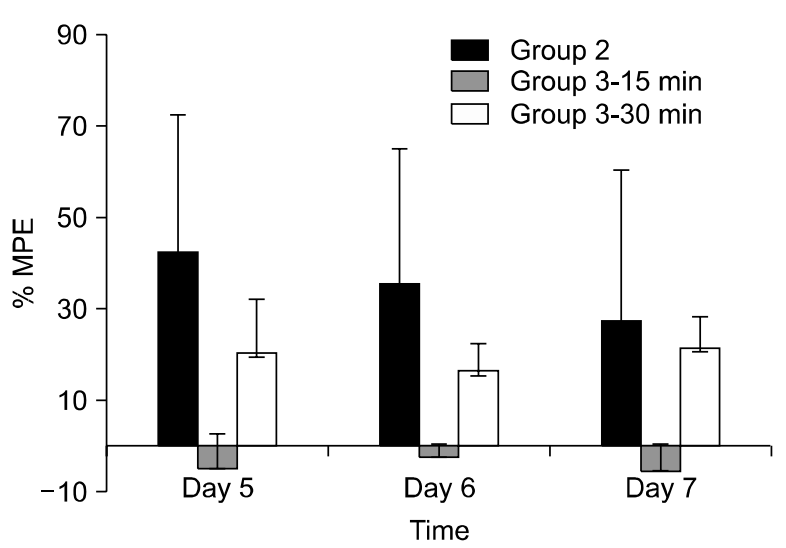

Fig. 3. Percentage of maximal possible effect (\%MPE) according to the groups. Group 2 showed the largest MPE at the $5^{\text {th }}$ day after SNL. Group 2, UTI 50,000 U/kg administration from the day of SNL for 3 days before neuropathic pain has evoked; Group 3-15 min, withdrawal thresholds were assessed 15 minutes after UTI injection, UTI $50,000 \mathrm{U} / \mathrm{kg}$ administration was started from the $5^{\text {th }}$ day after SNL, which is after neuropathic pain has evoked; Group 3-30 min, withdrawal thresholds were assessed 30 minutes after UTI injection, UTI 50,000 U/ kg administration was started from the $5^{\text {th }}$ day after SNL, which is after neuropathic pain has evoked; Day $5,5^{\text {th }}$ day after spinal nerve ligation; Day $6,6^{\text {th }}$ day after spinal nerve ligation; Day $7,7^{\text {th }}$ day after spinal nerve ligation. Values are mean \pm SD of withdrawal threshold. 
after 5 days. Administration of UTI 50,000 U/kg for 3 days after spinal nerve ligation, which was administered before neuropathic pain was evoked, increased the paw withdrawal threshold significantly $(P<0.05)$ (Fig. 2). Administration of UTI $50,000 \mathrm{U} / \mathrm{kg}$ starting from the $5^{\text {th }}$ day after spinal nerve ligation increased the paw withdrawal threshold after 30 minutes. However, there was no statistical significance when compared to the control group. The largest MPE was $44 \%$ in group 2 on the $5^{\text {th }}$ day compared to $21 \%$ in group 3 on the $7^{\text {th }}$ day (Fig. 3).

\section{DISCUSSION}

The main result of this study is that UTI administered right after SNL increased the paw withdrawal threshold. Despite the many good study designs and investigations, the mechanism of chronic neuropathic pain is still not completely understood because of its complexity [1]. Advanced studies showed that the pathogenesis of neuropathic pain consists of complex changes of neuronal systems, inflammatory immune responses, glial cells, inflammatory cytokines and so forth $[1,3]$. Under these circumstances, there have been efforts to explain neuropathic pain using the concept of neuro-immune disorder [1]. In this respect, nerve injury can cause an imbalance between pro-inflammatory and anti-inflammatory mechanisms, and this is one of the factors of NPS. Thus, promoting anti-inflammatory processes could prevent conversion of acute nociceptive pain to chronic neuropathic pain.

Ulinastatin is a serine protease inhibitor which is extensively used clinically in patients with pancreatitis, hemorrhage, shock, or undergoing surgery because of its anti-inflammatory and cytoprotective effects $[4,6-8]$. Such effects include inhibition of proteases, stabilizing lysosomal membranes, and inhibition of inflammatory cytokine production such as IL-6, IL-8, or TNF- $\alpha[6,12]$. Recently, its anti-inflammatory effects have focused on neuroprotection after brain injury caused by autoimmune myelitis or cerebral ischemia/reperfusion injury [7,8]. In addition, it has been known that activation of proteinase-activated receptors (PAR) play a role in neuronal sensitization [13]. Under inflammatory condition, serine protease shows a proteolytic activity, which initiates the signaling of PAR. Activation of PAR-2 signals, which are expressed in DRG neurons, has pro-inflammatory effects that play a role in the pathogenesis of inflammatory pain by mediating $\mathrm{pe}^{-}$ ripheral sensitization of nociceptors. Thus, we hypothesized that UTI, a serine protease inhibitor, also inhibits the inflammatory response after SNL which may attenuate the progress of NPS.

According to the literatures, activation of immune cells and inflammatory processes starts in the first 24 hours after nerve injury. Allodynia and hyperalgesia develop after 3 days, and thereafter, maintenance of allodynia and hyperalgesia starts [1]. A decrease in the withdrawal threshold occurs roughly 3 to 5 days after SNL [10]. Therefore, we administered UTI for 3 days after SNL in group 2 to identify the preventive effect of UTI against the development of NPS. Additionally, we also injected UTI starting from the $5^{\text {th }}$ day after SNL to determine if there were any pain reducing effects through the anti-inflammatory effect.

As mentioned earlier, when spinal nerve damage occurs, activation of microglia and astrocyte begins during the first 3 days after the damage, and algesic mediators, which enhance neuronal nociceptive transmission, and analgesic mediators, which decrease pain, are released by activated immune and immune-like glial cells [1]. During this stage, pro-inflammatory cytokines such as IL-1, IL-6, IL-10, and TNF have an important role in the development of neuropathic pain. If the balance between algesic mediators and analgesic mediators breaks down and pro-inflammatory cytokines become predominant, pain hypersensitivity and propagation of persistent pain states could occur and NPS may develop [1,2]. Lee et al. [14] have investigated the induction of mRNAs for pro-inflammatory cytokines in the dorsal root ganglia and spinal cord after spinal nerve injury. Their results showed that TNF- $\alpha$ and IL-1 $\beta$ have key roles during the initiation phase of persistent neuropathic pain, while $\mathrm{IL}-6$ has an important role during the maintenance phase.

In this view, UTI may have great advantages as an anti-inflammatory effect that inhibits pro-inflammatory cytokines production such as IL-6, IL-10, and TNF- $\alpha$ $[5,6,12]$. In this study, the paw withdrawal threshold, which was observed for 3 days starting from the $5^{\text {th }}$ day after SNL was significantly increased in the rats that were treated with UTI right after SNL. Whereas, when UTI was administrated starting from 5 days after SNL, the paw withdrawal threshold increased 30 minutes after injection to some degree but there was no statistical significance compared to the control group. The results suggest that UTI, administered right after SNL, has a protective effect 
against the development of allodynia and hyperalgesia. Such a protective effect is thought to be the result of the inhibition of IL- 6 and TNF- $\alpha$ which have roles in the initiation of persistent neuropathic pain. In addition, UTI that was administered in group 3 may have an analgesic effect after 30 min by inhibiting IL-6 $\alpha$ which has roles in its maintenance.

The absence of any actual measurements of pro-inflammatory cytokines or immunohistochemistry was a limitation of this study. However, we conducted this research as a preliminary study, and further evaluation such as responses according to the dosage, administration duration, measurement of cytokines, and long-term follow-up should be done.

In conclusion, ulinastatin, administered for 3 days after SNL, increased the paw withdrawal threshold and it could have a neuroprotective effect in the rat model of neuropathic pain.

\section{REFERENCES}

1. Austin PJ, Moalem-Taylor G. The neuro-immune balance in neuropathic pain: involvement of inflammatory immune cells, immune-like glial cells and cytokines. J Neuroimmunol 2010; 229: 26-50.

2. Üçeyler N, Sommer C. Cytokine regulation in animal models of neuropathic pain and in human diseases. Neurosci Lett 2008; 437: 194-8.

3. Thacker MA, Clark AK, Marchand F, McMahon SB. Pathophysiology of peripheral neuropathic pain: immune cells and molecules. Anesth Analg 2007; 105: 838-47.

4. Park KH, Lee KH, Kim H, Hwang SO. The anti-inflammatory effects of ulinastatin in trauma patients with hemorrhagic shock. J Korean Med Sci 2010; 25: 128-34.
5. Aosasa S, Ono S, Mochizuki H, Tsujimoto $H$, Ueno C, Matsumoto A. Mechanism of the inhibitory effect of protease inhibitor on tumor necrosis factor alpha production of monocytes. Shock 2001; 15: 101-5.

6. Park JH, Kwak SH, Jeong CW, Bae HB, Kim SJ. Effect of ulinastatin on cytokine reaction during gastrectomy. Korean J Anesthesiol 2010; 58: 334-7.

7. Cao LJ, Wang J, Hao PP, Sun CL, Chen YG. Effects of ulinastatin, a urinary trypsin inhibitor, on synaptic plasticity and spatial memory in a rat model of cerebral ischemia/ reperfusion injury. Chin J Physiol 2011; 54: 435-42.

8. Shu Y, Yang Y, Qiu W, Lu Z, Li Y, Bao J, et al. Neuroprotection by ulinastatin in experimental autoimmune encephalomyelitis. Neurochem Res 2011; 36: 1969-77.

9. Kim SH, Chung JM. An experimental model for peripheral neuropathy produced by segmental spinal nerve ligation in the rat. Pain 1992; 50: 355-63.

10. Chung JM, Kim HK, Chung K. Segmental spinal nerve ligation model of neuropathic pain. Methods Mol Med 2004; 99: $35-45$.

11. Dogrul A, Ossipov MH, Lai J, Malan TP Jr, Porreca F. Peripheral and spinal antihyperalgesic activity of SIB-1757, a metabotropic glutamate receptor (mGLUR(5)) antagonist, in experimental neuropathic pain in rats. Neurosci Lett 2000; 292: $115-8$.

12. Wang $X$, Xue Q, Yan F, Li L, Liu J, Li S, et al. Ulinastatin as a neuroprotective and anti-inflammatory agent in infant piglets model undergoing surgery on hypothermic low-flow cardiopulmonary bypass. Paediatr Anaesth 2013; 23: 209-16.

13. Zhu WJ, Yamanaka H, Obata K, Dai Y, Kobayashi K, Kozai $T$, et al. Expression of mRNA for four subtypes of the proteinase-activated receptor in rat dorsal root ganglia. Brain Res 2005; 1041: 205-11.

14. Lee HL, Lee KM, Son SJ, Hwang SH, Cho HJ. Temporal expression of cytokines and their receptors mRNAs in a neuropathic pain model. Neuroreport 2004; 15: 2807-11. 